\title{
The Authors Reply: Effects of Age, Period, and Cohort on Health Status in Korean Baby Boom Generation
}

Thank you for your interest and valuable comments in our study. I completely agree with the 2 points of contention pointed out in your letter. I believe that your comments and opinions are based on a full understanding of our study findings. In response to the first comment about separating the age effect and cohort effect being a mandatory process for specifically analyzing and interpreting the health status of Baby Boomers, we did not adjust for age because we considered that the interaction between age and cohorts divided by birth year might have been high. Match samples via birth year and age may be one of effective solutions to compare age effect and cohort effect.

In addition, I would like to suggest age-period-cohort (APC) model for a future study focusing on distinguish the effect of age, period, and cohort of generations. This method is a technique used in epidemiological studies ${ }^{1,2)}$ to examine the time-related effects of age, period, and cohort separately. Age effects record effects on different age groups over time, while period effects represent the effects on all age groups simultaneously within the same period. Finally, cohort effects go beyond particular groups to focus on effects shared by groups with the same birth years. Age effects primarily reflect biological changes, whereas period and cohort effects reflect environmental changes. In particular, cohort effects involve the influence of early environmental exposure being expressed as a specific health risk for a specific cohort. The APC model is used with the assumption that age, period, and cohort share a linear dependency. To resolve the problem of not being able to separate the major related factors within a standard, fully applied APC model, a general method of estimating the setting constraints on the parameters has been used ${ }^{3)}$. Independent variables (health outcomes, such as prevalence etc.) can be log transformed and the estimated values can be calculated using the model shown below.

$$
\phi_{i j k}=\mu+\alpha_{i} \text { Age }+\beta_{j} \text { Period }+\gamma_{k} \text { Cohort }+\epsilon_{i j k}
$$

where, $\phi_{i j k}$ is the natural logarithm of the prevalence of a specific health-related outcome; $\mu$ is the intercept term; $\alpha_{i}$ is the effect of age $\mathrm{l}_{i} \beta_{j}$ is the effect of period $\mathrm{j} ; \gamma_{k}$ and is the effect of cohort $k$. If the analysis is performed using this method, the problem of separating the effects of age and cohorts in our study can be resolved and in addition, a deeper understanding can be gained into the difference and diversity of health levels among future elderly generations. Moreover, further studies on the causes of health differences, and in identifying increases or decreases in relevant causal factors, would be feasible using this method.

We also completely agree with your second comment and suggestion on the interpretation of depressive symptoms among baby boomers. As indicated in the Introduction section of our study, it has often been noted that baby boomers in Korea have a higher education level than those from previous generations and that they have lived in relative affluence in their younger years, but that in their middle-age years they also face financial burdens and increased pressure as a 'sandwich' generation in fulfilling simultaneous roles caring for both their children and their elderly parents ${ }^{4,5)}$. Annual trends regarding depressive symptoms from 20092013 medical panel data indicate a prevalence level of depressive symptoms similar to that of the younger generation in 2009, whereas by 2013 the prevalence of depressive symptoms had increased to a level similar to the elderly generation. An overall high level of depressive symptoms within the cohort identified as baby boomers is an important outcome, which should be thoroughly investigated. Furthermore, a rapid increase in depressive symptoms over time compared to other generations indicates the possible impact of psychosocial influence, and these effects continue to increase as the baby boomer cohort shows the age effect of becoming older generation. More targeted studies are needed to study the mental health of baby boomers, while specific social and policy considerations in respect of this cohort are also deemed necessary.

Conflicts of Interest Disclosures: The researcher claims no conflicts of interest. 


\section{Soong-Nang Jang}

Nursing Science Research Institute and Red Cross College of Nursing, Chung-Ang University, Seoul, Korea

Corresponding Author: Soong-Nang Jang, RN, MPH, PhD Red Cross College of Nursing, Chung-Ang University, 84 Heukseok-ro, Dongjak-gu, Seoul 06974, Korea

Tel: +82-2-820-5806, Fax: +82-2-824-7967

E-mail: sjang@cau.ac.kr

Received: June 6, 2017

Revised: June 6, 2017

Accepted: June 6, 2017

\section{REFERENCES}

1. Jee SH, Kim IS, Suh I, Shin D, Appel LJ. Projected mortality from lung cancer in South Korea, 1980-2004. Int J Epidemiol 1998;27:365-9.

2. Osmond C, Gardner MJ. Age, period and cohort models applied to cancer mortality rates. Stat Med 1982;1:245-59.

3. Holford TR. The estimation of age, period and cohort effects for vital rates. Biometrics 1983;39:311-24.

4. Jung KH, Son CK, Park BM. Characteristics and policies for new older generation, Sejong: Korean Institute of Health and Social Affairs; 2010.

5. Jung $\mathrm{KH}$, Policy directin for retirement and aging among babyboom generation. Ministry of Health and Welfare, Sejong: Korean Institute of Health and Social Affairs; 2011. 